\title{
Plasma transforming growth factor $\beta 1$ as a biochemical marker to predict the persistence of atrial fibrillation after the surgical maze procedure
}

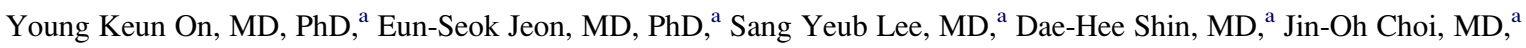 \\ Jidong Sung, MD, MPH, ${ }^{\mathrm{a}}$ June Soo Kim, MD, PhD, ${ }^{\mathrm{a}}$ Kiick Sung, MD, ${ }^{\mathrm{b}}$ and PyoWon Park, MD, PhD ${ }^{\mathrm{b}}$
}

\begin{abstract}
Objectives: The Cox maze procedure was developed as a surgical treatment for atrial fibrillation. However, atrial fibrillation recurs in some patients, and atrial remodeling in the form of fibrosis can lead to perpetuation of atrial fibrillation. To identify the predictor of the persistence of atrial fibrillation after the maze procedure using cryoablation, we evaluated the preoperative plasma transforming growth factor $\beta 1$. We also examined the correlations between plasma transforming growth factor $\beta 1$ levels and the degree of atrial fibrosis.
\end{abstract}

Methods: Preoperative plasma transforming growth factor $\beta 1$ levels were measured in 86 consecutive patients (age, $54 \pm 12$ years) who underwent both the open heart operation for valvular heart disease and the surgical maze procedure with cryoablation for persistent atrial fibrillation. We measured the degree of fibrosis from the tissue of the left atrium.

Results: At 1 year's follow-up, 10 of 86 patients had persistent atrial fibrillation. Patients with persistent atrial fibrillation had higher preoperative plasma transforming growth factor $\beta 1$ levels than the patients with sinus rhythm $(0.44 \pm 0.29$ vs $0.32 \pm 0.15 \mathrm{ng} / \mathrm{mL}, P=.03)$. Patients with persistent atrial fibrillation had higher mRNA expressions of collagen III and lower mRNA expressions of atrial natriuretic peptide than those with sinus rhythm, and the plasma transforming growth factor $\beta 1$ levels correlated with the degree of fibrosis in the left atrium $(r=0.497, P=.022)$. Multiple logistic regression analysis revealed that plasma transforming growth factor $\beta 1$ levels were independently associated with the postoperative persistence of atrial fibrillation at 1 year's follow-up.

Conclusions: Preoperative plasma transforming growth factor $\beta 1$ levels could be used to predict the persistence of atrial fibrillation at 1 year's follow-up after the surgical maze procedure by using cryoablation. Preoperative plasma transforming growth factor $\beta 1$ levels were correlated with the degree of fibrosis in the left atria of patients with mitral valvular heart disease.

The Cox maze procedure was developed as a surgical treatment of atrial fibrillation (AF), which aims to eliminate anatomically determined re-entrant circuits by creating contiguous lines of scar tissue between the pulmonary veins and the mitral valve annulus. ${ }^{1}$ Restoration of sinus rhythm (SR) has been reported to be successful in $75 \%$ to $95 \%$ of patients undergoing the Cox maze procedure. ${ }^{2-4}$

Atrial remodeling leads to perpetuation of AF. Structural remodeling in the form of fibrosis alters the substrate. Therefore the success rate of the maze operation might be related to the degree of atrial fibrosis. Among many echocardiographic parameters and biomarkers, left atrial dimension in echocardiographic analysis, AF duration, ${ }^{5,6}$ plasma atrial

\footnotetext{
From the Division of Cardiology, Department of Medicine, ${ }^{\mathrm{a}}$ and the Department of Thoracic and Cardiovascular Surgery, ${ }^{\mathrm{b}}$ Cardiac and Vascular Center, Samsung Medical Center, Sungkyunkwan University School of Medicine, Seoul, Korea.

Received for publication July 10, 2008; revisions received Sept 17, 2008; accepted for publication Oct 16, 2008.

Address for reprints: Eun-Seok Jeon, MD, PhD, Division of Cardiology, Department of Medicine, Cardiac and Vascular Center, Samsung Medical Center, Sungkyunkwan University School of Medicine, 50 Irwon-dong, Kangnam-gu, 135-710, Seoul, Korea (E-mail: eunseokjeon@samsung.com).

J Thorac Cardiovasc Surg 2009;137:1515-20

$0022-5223 / \$ 36.00$

Copyright (c) 2009 by The American Association for Thoracic Surgery

doi:10.1016/j.jtcvs.2008.10.022
}

natriuretic peptide (ANP) level, ${ }^{7}$ and mRNA expression of a few cytokines, such as transforming growth factor (TGF) $\beta 1$, collagen I, and collagen III, have been reported as predictors of the outcome of the maze procedure.

To identify the predictors of the persistence of AF after the maze procedure with cryoablation, we evaluated the role of preoperative plasma TGF- $\beta 1$ and evaluated the degree of atrial fibrosis in histology in patients who underwent both mitral valvular surgery and the maze procedure with cryoablation because of persistent AF. We also examined the correlations between plasma TGF- $\beta 1$ levels and the degree of atrial fibrosis in the left atrium.

\section{MATERIALS AND METHODS \\ Patients}

The trial was conducted between March 2005 and December 2006 in the Departments of Cardiology and Thoracic and Cardiovascular Surgery, Cardiovascular Center, Samsung Medical Center, Seoul, South Korea. Patients requiring mitral valve surgery who also had a history of persistent $\mathrm{AF}$ were eligible. The term longstanding persistent $A F$ denotes the presence of uninterrupted $\mathrm{AF}$ for at least 6 months that showed no evidence of spontaneous reversibility to SR. Patients with sick sinus syndrome, uncontrolled hyperthyroidism, a permanent pacemaker, or previous cardiac surgery were excluded. The protocol was approved by the institutional research ethics committee. Written informed consent was obtained from all participants, 


$$
\begin{aligned}
& \text { Abbreviations and Acronyms } \\
& \begin{aligned}
\text { AF } & =\text { atrial fibrillation } \\
\text { ANP } & =\text { atrial natriuretic peptide } \\
\text { ECG } & =\text { electrocardiogram } \\
\text { ELISA } & =\text { enzyme-linked immunosorbent assay } \\
\text { MMP } & =\text { matrix metalloproteinase } \\
\text { SR } & =\text { sinus rhythm } \\
\text { TGF } & =\text { transforming growth factor }
\end{aligned}
\end{aligned}
$$

and the recommendations of the revised version of the Declaration of Helsinki were met.

Eighty-six consecutive patients (age, $54 \pm 12$ years; 37 male and 49 female patients) who underwent both the open heart operation for mitral valvular heart disease and the surgical maze procedure for AF were enrolled. The mean duration of AF before the surgical maze procedure was 3.4 years (approximately 6 months to 15 years). The underlying mitral valvular heart diseases were rheumatic heart disease in 58 patients and degenerative mitral valvular disease in 28 patients.

The primary end point of the study was the persistence of AF during the 12-month follow-up period after the maze procedure with cryoablation.

\section{Operations and the Maze Procedure}

Operations were performed between March 2005 and December 2006. All procedures were performed through a median sternotomy by using cardiopulmonary bypass with bicaval venous drainage during moderate hypothermia on an arrested heart. When performing a valve operation, the valve was excised first, and then the maze procedure was performed, followed by valve replacement or reconstruction. We simplified the procedure. Neither atrial appendage was excised. Maze lesions were made by means of cryoablation. Cryoablation lesions were created endocardially with a T-shaped Cryoprobe (EP Technologies, Boston Scientific Corp, San Jose, Calif). Cryogenerators were set at $-60^{\circ} \mathrm{C}$. Each cryoablation was delivered for $160 \mathrm{sec}-$ onds to achieve transmural lesions, as previously described. ${ }^{2}$

These lesions were made to isolate the pulmonary veins with the posterior left atrial wall in conjunction with the left atriotomy incision by encircling them all in one and also connecting the isolated area by creating lines to the mitral annulus. A right-sided cryoablation lesion was applied at the inferior vena cava to the coronary sinus from the tricuspid annulus to the interatrial groove. If the right atrium was large or if the tricuspid valve incompetence was significant, another right-sided lesion was made from the right atrial appendage to the tricuspid annulus. We did not place a lesion from the superior vena cava to the inferior vena cava. Then the left atrial appendage was over sewn from within the left atrium.

\section{Blood and Tissue Sampling}

Blood samples were obtained from peripheral veins at the day of the operation. After centrifugation, the isolated plasma was stored before enzymelinked immunosorbent assay (ELISA). Tissue samples were randomly taken just from the areas surrounding the left atriotomy from the left atrium at the time of mitral valve surgery. A part of the tissue samples was processed for pathology, and another part was immediately frozen in liquid nitrogen and stored at $-80^{\circ} \mathrm{C}$.

\section{Postoperative Follow-up}

Cardiac rhythm was continuously monitored for 48 hours. Thereafter, daily 12-lead electrocardiograms (ECGs) were obtained during hospitalization. Per protocol, all patients stopped antiarrhythmic medication after the maze operation with cryoablation. In patients who remained in AF after the operation, electrical cardioversion was attempted. Warfarin was admin- istered to all patients. Before hospital discharge, all patients underwent transthoracic echocardiographic analysis. All patients were followed up at $1,3,6,9$, and 12 postoperative months. They had a clinical examination and a 12-lead ECG. At 6 and 12 months, 24-hour Holter monitoring and transthoracic echocardiographic analysis were also carried out. During follow-up, if a patient reported symptoms suggestive of arrhythmia either at a specified visit or between visits, 24-hour Holter monitoring was performed (and an ECG was obtained, if appropriate), and if AF was observed, the patient was deemed to have reverted to $\mathrm{AF}$ at the next formal assessment. Likewise, the findings on any sporadic ECGs obtained by the patients' physicians were similarly taken into account. At 1 year's follow-up after the maze procedure, 56 patients had maintained the restored SR, and 10 patients had persistent AF despite medical and electrocardioversion. Twenty patients showed recurrent $\mathrm{AF}$, which was converted to SR after application of amiodarone.

Measurements of plasma cytokines levels. The levels of plasma N-terminal prohormone brain-type natriuretic peptide, high-sensitivity C-reactive protein, ANP, tissue inhibitor of metalloproteinase, TGF$\beta 1$, matrix metalloproteinase (MMP) 3, and pro-MMP-1 were measured by means of ELISA with a Quantikine kit (R\&D Systems, Inc, Minneapolis, Minn). All samples were measured with the same batch of ELISA kits.

mRNA expressions of cytokines in left atrial tissue. A part of the resected left atrial tissues were frozen in liquid nitrogen and stored at $-80^{\circ} \mathrm{C}$ for Northern blot analysis to obtain molecular biologic evidence regarding the atrial qualitative differences between the $\mathrm{AF}$ and $\mathrm{SR}$ groups (SR group, $\mathrm{n}=56 ; \mathrm{AF} \rightarrow \mathrm{SR}$ group, $\mathrm{n}=20$; $\mathrm{AF}$ group, $\mathrm{n}=10$ ). Total RNA was extracted with TRIzol reagent (Gibco BRL, Carlsbad, Calif) and DNA was transcripted with ThermoScript reverse transcriptase-polymerase chain reaction systems (Gibco BRL). The expression of mRNA was semiquantified by using an internal standard of glyceraldehyde-3-phosphate dehydrogenase.

\section{Histology}

Half of the resected left atrial samples were fixed in $6 \%$ buffered formalin, embedded in paraffin, and stained with hematoxylin and eosin for routine histologic examination. Sections $(5 \mu \mathrm{m})$ were stained with Masson trichrome and Picro Sirius red for quantification of fibrosis. Sirius redstained slides were scanned, and the percentage of fibrosis was automatically calculated with 5 random fields of each slide at the same magnification $(\times 400)$ in 22 randomly selected patients by using the Image Pro Plus program (Media Cybernetics, Inc, Bethesda, Md).

\section{Statistical Analysis}

Summary values are presented as means \pm standard deviations. Categorical variables were compared with the $\chi^{2}$ test or Fisher's exact test, and continuous variables were compared with an unpaired $t$ test or Mann-Whitney test as appropriate. Correlation analysis was done with the Pearson correlation test.

Predictors of AF persistence were identified with univariate and multiple logistic regression analysis. First, a series of variables were screened with univariate analysis. Then the variables that attained a $P$ value of less than .10 were entered into a multiple logistic regression model. Statistical analysis was performed with SAS version 9.1 software (SAS Institute, Inc, Cary, NC).

\section{RESULTS}

At 1 year's follow-up after the maze procedure, 56 of 86 consecutive patients had maintained the restored SR (SR group, $\mathrm{n}=56$ ), and 10 patients had persistent AF despite medical and electrocardioversion (AF group, $\mathrm{n}=10$ ). Twenty patients showed recurrent $\mathrm{AF}$, which was converted to $\mathrm{SR}$ after medication $(\mathrm{AF} \rightarrow \mathrm{SR}$ group, $\mathrm{n}=20$ ). The clinical characteristics of patients are summarized in 
TABLE 1. Clinical characteristics of patients

\begin{tabular}{lcccc}
\hline & SR & AF $\rightarrow$ SR & AF & P value \\
\hline No. & 56 & 20 & 10 & $57.5 \pm 13.0$ \\
Age (y) & $52.5 \pm 10.2$ & $57.8 \pm 14.3$ & $3 / 7$ & .143 \\
Sex (M/F) & $26 / 30$ & $8 / 12$ & $3(30 \%)$ & .597 \\
Hypertension & $11(20 \%)$ & $6(30 \%)$ & 0 & .556 \\
DM & $7(12 \%)$ & $4(20 \%)$ & 0 & .301 \\
Stroke/TIA & $6(11 \%)$ & $2(10 \%)$ & $3(30 \%)$ & $7 / 3$ \\
Smoking & $13(23 \%)$ & $5(25 \%)$ & .557 \\
Rheum/Degen & $38 / 18$ & $13 / 7$ & $4.8 \pm 5.2$ & .957 \\
AF duration (y) & $3.2 \pm 4.2$ & $3.3 \pm 3.1$ & .499 \\
\hline
\end{tabular}

At 1 year's follow-up after the maze procedure, 56 of 86 consecutive patients had maintained restored sinus rhythm (SR group, $\mathrm{n}=56)$, and 10 patients had persistent atrial fibrillation despite medical and electrocardioversion ( $\mathrm{AF}$ group, $\mathrm{n}=10$ ). Twenty patients showed recurrent atrial fibrillation, which was converted to sinus rhythm after medication $(\mathrm{AF} \rightarrow \mathrm{SR}$ group, $\mathrm{n}=20$ ). $P$ values were determined by means of analysis of variance. $S R$, Sinus rhythm group; $A F \rightarrow S R$, atrial fibrillation to sinus rhythm group; $A F$, atrial fibrillation group; $M$, Male; $F$, female; $D M$, diabetes mellitus; TIA, transient ischemic attack; Rheum, rheumatic heart disease; Degen, degenerative heart disease.

Table 1. The prevalence of arterial hypertension, diabetes mellitus, stroke, or transient ischemic attack was not significantly different among groups. The duration of AF looked similar $(P=.499)$. The type of underlying valvular heart disease, such as rheumatic or degenerative disease, was not different among groups.

Among the echocardiographic parameters, the preoperative left atrial volume index was larger in the AF group than in the SR group $\left(88.6 \pm 27.6\right.$ vs $118.5 \pm 51.6 \mathrm{~mm}^{3}$, $P<.05)$. Immediate postoperative left atrial diameters were larger in the AF group than in the SR group (50.0 \pm 6.1 vs $53.7 \pm 8.7 \mathrm{~mm}, P<.05$, Table 2 ).

The AF group had higher plasma TGF- $\beta 1$ levels than the SR group $(0.44 \pm 0.29$ vs $0.32 \pm 0.15 \mathrm{ng} / \mathrm{mL}, P<.05$, Table 3).

The mRNA expressions of collagen III $\alpha(0.21 \pm 0.20$ vs $0.12 \pm 0.12, P=.061$ compared with internal standard glyceraldehyde-3-phosphate dehydrogenase by means of reverse transcriptase-polymerase chain reaction) were higher in $\mathrm{AF}$ group, and the mRNA expressions of ANP were lower ( 0.31 \pm 0.16 vs $0.60 \pm 0.76, P=.013)$ in the AF group than in the SR group (Tables 4 and 5).
Multiple logistic regression analysis revealed that plasma TGF- $\beta 1$ levels were independently associated with the postoperative persistence of $\mathrm{AF}$ at 1 year's follow-up after the surgical maze procedure (Table 6).

In Masson trichrome and Sirius red staining, more fibrosis was identified in the AF group. The degree of fibrosis was correlated with the echocardiographic left atrial diameter, left atrial volume index, and preoperative plasma TGF- $\beta 1$ level (Figures 1 and 2).

Plasma TGF- $\beta 1$ levels of greater than $0.30 \mathrm{ng} / \mathrm{mL}$ had a sensitivity of $70.0 \%$ and a specificity of $60.8 \%$ for the postoperative persistence of $\mathrm{AF}$ at 1 year's follow-up after the surgical maze procedure with cryoablation.

\section{DISCUSSION}

The importance of fibrosis appears to be increased in patients with persistent AF. Atrial fibrosis provides a pathophysiologic substrate for postoperative AF (ie, arrhythmogenic substrate for AF). ${ }^{9,10}$

Patients with chronic AF were shown to have higher levels of myocardial interstitial fibrosis compared with control subjects, who are known to be arrhythmogenic. ${ }^{1-13}$

TABLE 2. Echocardiographic parameters of the patients

\begin{tabular}{|c|c|c|c|c|}
\hline & SR & $\mathbf{A F} \rightarrow \mathbf{S R}$ & $\mathbf{A F}$ & $P$ value \\
\hline No. & 56 & 20 & 10 & \\
\hline \multicolumn{5}{|c|}{$\begin{array}{l}\text { Preoperative echocardiographic } \\
\text { analysis }\end{array}$} \\
\hline LAD (mm) & $59.1 \pm 7.1$ & $63.1 \pm 7.9$ & $63.2 \pm 7.5$ & .060 \\
\hline LAVI $\left(\mathrm{mm}^{3}\right)$ & $88.6 \pm 27.6$ & $102.4 \pm 30.8$ & $118.5 \pm 51.6^{*}$ & .021 \\
\hline \multicolumn{5}{|c|}{$\begin{array}{l}\text { Postoperative echocardiographic } \\
\text { analysis (before discharge) }\end{array}$} \\
\hline $\mathrm{LAD}(\mathrm{mm})$ & $50.0 \pm 6.1$ & $52.9 \pm 5.7$ & $53.7 \pm 8.7^{*}$ & .044 \\
\hline LAVI $\left(\mathrm{mm}^{3}\right)$ & $57.9 \pm 15.1$ & $63.5 \pm 15.7$ & $68.1 \pm 19.2$ & .127 \\
\hline
\end{tabular}


TABLE 3. Biochemical markers among groups

\begin{tabular}{|c|c|c|c|c|}
\hline & SR & $\mathbf{A F} \rightarrow \mathbf{S R}$ & $\mathbf{A F}$ & $P$ value \\
\hline No. & 56 & 20 & 10 & \\
\hline NT-proBNP (pg/mL) & $1849 \pm 3959$ & $1991 \pm 2347$ & $1154 \pm 905$ & .807 \\
\hline hsCRP (mg/dL) & $0.37 \pm 1.50$ & $0.50 \pm 1.34$ & $0.20 \pm 0.21$ & .857 \\
\hline ANP (ng/mL) & $6.32 \pm 6.19$ & $5.16 \pm 2.91$ & $3.97 \pm 1.65$ & .367 \\
\hline TIMP-1 (ng/mL) & $1.32 \pm 0.72$ & $1.38 \pm 0.75$ & $1.35 \pm 0.81$ & .951 \\
\hline TGF- $\beta 1$ (ng/mL) & $0.32 \pm 0.15$ & $0.27 \pm 0.11$ & $0.44 \pm 0.29 *$ & .030 \\
\hline MMP-3 (ng/mL) & $1.10 \pm 0.94$ & $0.87 \pm 0.45$ & $0.78 \pm 0.45$ & .359 \\
\hline Pro-MMP-1 (ng/mL) & $1.58 \pm 1.26$ & $1.50 \pm 1.66$ & $1.34 \pm 0.81$ & .861 \\
\hline
\end{tabular}

Preoperative cytokines were measured by means of enzyme-linked immunosorbent assay. Plasma transforming growth factor $\beta 1$ levels were increased in the AF group. $P$ values were determined by means of analysis of variance. $S R$, Sinus rhythm group; $A F \rightarrow S R$, atrial fibrillation to sinus rhythm group; $A F$, atrial fibrillation group; $N T$ - $p r o B N P$, N-terminal prohormone brain natriuretic peptide; $h S C R P$, high-sensitivity C-reactive protein; $A N P$, atrial natriuretic protein; $T I M P$, tissue inhibitor of metalloproteinase; $M M P$, matrix metalloproteinase; TGF- $\beta 1$, Transforming growth factor $\beta 1 .{ }^{*} P<.05$ compared with the SR group.

The precise mechanisms and signaling pathways involved in the development of atrial fibrosis are unknown. It appears that the atrium is more susceptible to atrial fibrosis than is the ventricle, and currently, 3 interrelated pathways appear to be involved: the renin-angiotensin system, TGF- $\beta 1$, and the oxidative stress pathways. ${ }^{12}$

Fibrosis is thought to be partially mediated by TGF- $\beta 1$, a potent stimulator of collagen-producing cardiac fibroblasts. TGF- $\beta 1$ is a profibrotic cytokine that stimulates the production of extracellular matrix proteins in organs. Furthermore, in animals overexpression of TGF- $\beta$ results in tissue fibrosis and organ dysfunction. The pathogenic effects of TGF- $\beta 1$ have now been suggested to play a major role in valvular disease and arrhythmia, particularly AF. In a transgenic mouse model ${ }^{13}$ constitutional activation of TGF- $\beta 1$ produces atrial restricted fibrosis and promotes $\mathrm{AF}$ inducibility. TGF- $\beta 1$-associated fibrosis results in an inhomogeneous substrate for electrical propagation. This environment impedes anisotropic or linear conduction leading to the development of arrhythmia. ${ }^{13-15}$ TGF- $\beta 1$ has been shown to increase connective tissue growth factor synthesis within different fibroblast subtypes, including cardiac fibroblasts, most probably by acting on TGF- $\beta 1$ response elements localized in the connective tissue growth factor promoter site. ${ }^{16}$
Homeostasis of the extracellular matrix is a balance between synthesis and degradation. Proteolytic enzymes, such as MMPs, play an important role in promoting change and remodeling. ${ }^{15,17}$ By breaking down the extracellular matrix, MMPs contribute to matrix turnover, to dilatation, and to structural remodeling. The roles of TGF- $\beta 1$ and MMPs in cardiac remodeling might be intertwined, which allows for the local production of extracellular proteins and increased fibrosis within the myocardium of the heart associated with cardiac remodeling.

Myocardial fibrosis plays an important role in predisposition to arrhythmia. ${ }^{11}$ Mice with increased expression of TGF- $\beta 1$ were prone to AF as a result of increased levels of atrial fibrosis. ${ }^{13}$ TGF- $\beta 1$ plays an important role within the "AF begets AF"' remodeling process.

Patients with AF display increased atrial fibrous tissue content, along with increased expression of collagen I and III. ${ }^{18}$ The composition of the extracellular matrix might provide a substrate for the initiation of postoperative AF. ${ }^{19,20}$

The plasma ANP level might reflect atrial degenerative change and might predict the outcome of the maze procedure. Yoshihara and colleagues ${ }^{7}$ reported that the preoperative plasma ANP level was significantly lower in the AF group than in the SR group. The mRNA expression of ANP in the left atrial appendage tissues was also

TABLE 4. mRNA expressions of cytokines in left atrial tissue among groups

\begin{tabular}{lcccc}
\hline & SR & AF $\rightarrow$ SR & AF & P value \\
\hline No. & 56 & 20 & 10 & .396 \\
ANP & $0.60 \pm 0.76$ & $0.48 \pm 0.49$ & $0.31 \pm 0.16^{*}$ & .520 \\
BNP & $0.14 \pm 0.24$ & $0.09 \pm 0.13$ & $0.17 \pm 0.18$ & .905 \\
TGF- $\beta$ 1 & $0.19 \pm 0.23$ & $0.19 \pm 0.17$ & $0.22 \pm 0.18$ & .600 \\
CTGF & $0.23 \pm 0.23$ & $0.28 \pm 0.25$ & $0.19 \pm 0.21$ & .666 \\
Collagen I $\alpha$ & $0.12 \pm 0.15$ & $0.12 \pm 0.11$ & $0.17 \pm 0.21$ & .183 \\
Collagen III $\alpha$ & $0.12 \pm 0.12$ & $0.14 \pm 0.15$ & $0.21 \pm 0.20$ & \\
\hline
\end{tabular}

The internal standard was glyceraldehyde-3-phosphate dehydrogenase. mRNA expressions were not different among groups. $P$ values were determined by means of analysis of variance. $S R$, Sinus rhythm group; $A F \rightarrow S R$, atrial fibrillation to sinus rhythm group; $A F$, atrial fibrillation group; $A N P$, atrial natriuretic peptide; $B N P$, brain natriuretic peptide; $T G F-\beta 1$, transforming growth factor $\beta 1 ; C T G F$, connective tissue growth factor. $* P<.05$ compared with the SR group. 
TABLE 5. Comparisons of echocardiographic and biochemical markers to predict the persistence of atrial fibrillation at 1 year's follow-up in the AF and SR groups

\begin{tabular}{lccc}
\hline & SR & AF & $P$ value \\
\hline No. & 56 & 10 & \\
Age (y) & $52.5 \pm 10.2$ & $57.5 \pm 13.0$ & .173 \\
AF duration (y) & $3.2 \pm 4.2$ & $4.8 \pm 5.2$ & .270 \\
Preoperative & & & \\
$\quad$ echocardiography & & & \\
LAD (mm) & $59.1 \pm 7.1$ & $63.2 \pm 7.5$ & .101 \\
LAVI (mm $\left.{ }^{3}\right)$ & $88.6 \pm 27.6$ & $118.5 \pm 51.6$ & .012 \\
Postoperative & & & \\
$\quad$ echocardiography & & & \\
LAD (mm) & $50.0 \pm 6.1$ & $53.7 \pm 8.7$ & .068 \\
LAVI (mm $\left.{ }^{3}\right)$ & $57.9 \pm 15.1$ & $68.1 \pm 19.2$ & .077 \\
Biologic markers & & & \\
NT-proBNP (pg/mL) & $1849 \pm 3959$ & $1154 \pm 905$ & .585 \\
hsCRP (mg/dL) & $0.37 \pm 1.50$ & $0.20 \pm 0.21$ & .724 \\
ANP (ng/mL) & $6.32 \pm 6.19$ & $3.97 \pm 1.65$ & .240 \\
TIMP-1 (ng/mL) & $1.32 \pm 0.72$ & $1.35 \pm 0.81$ & .901 \\
TGF- $\beta 1(\mathrm{ng} / \mathrm{mL})$ & $0.32 \pm 0.15$ & $0.44 \pm 0.29$ & .055 \\
MMP-3 (ng/mL) & $1.10 \pm 0.94$ & $0.78 \pm 0.45$ & .301 \\
Pro-MMP-1 (ng/mL) & $1.58 \pm 1.26$ & $1.34 \pm 0.81$ & .559 \\
mRNA expression & & & \\
ANP & $0.60 \pm 0.76$ & $0.31 \pm 0.16$ & .013 \\
BNP & $0.14 \pm 0.24$ & $0.17 \pm 0.18$ & .633 \\
TGF- $\beta 1$ & $0.19 \pm 0.23$ & $0.22 \pm 0.18$ & .678 \\
CTGF & $0.23 \pm 0.23$ & $0.19 \pm 0.21$ & .556 \\
Collagen I $\alpha$ & $0.12 \pm 0.15$ & $0.17 \pm 0.21$ & .444 \\
Collagen III $\alpha$ & $0.12 \pm 0.12$ & $0.21 \pm 0.20$ & .061 \\
\hline
\end{tabular}

$\overline{S R}$, Sinus rhythm group; $A F$, atrial fibrillation group; $L A D$, left atrial diameter; $L A V I$, left atrial volume index; $N T$-proBNP, N-terminal prohormone brain natriuretic peptide; $h S C R P$, high-sensitivity C-reactive protein; $A N P$, atrial natriuretic peptide; TIMP-1, tissue inhibitor of metalloproteinase $1 ; T G F-\beta 1$, transforming growth factor $\beta 1 ; M M P$, matrix metalloproteinase; $C T G F$, connective tissue growth factor.
TABLE 6. Biomarkers to predict persistent atrial fibrillation 1 year after the maze operation

\begin{tabular}{lrc}
\hline & B & $\boldsymbol{P}$ value \\
\hline Preoperative echocardiography & & \\
$\quad$ LAD $(\mathrm{mm})$ & -0.406 & .108 \\
LAVI $\left(\mathrm{mm}^{3}\right)$ & 0.078 & .071 \\
Postoperative echocardiography & & \\
LAD $(\mathrm{mm})$ & 0.339 & .135 \\
LAVI $\left(\mathrm{mm}^{3}\right)$ & -0.016 & .760 \\
TGF- $\beta 1(\mathrm{ng} / \mathrm{mL})$ & 10.934 & .042 \\
ANP & -4.857 & .108 \\
Collagen $\mathrm{I} \alpha$ & -8.616 & .229 \\
Collagen $\mathrm{III} \alpha$ & 12.890 & .126 \\
\hline Preop
\end{tabular}

Preoperative plasma levels of transforming growth factor $\beta 1$ could predict the persistency of atrial fibrillation in multiple logistic regression analysis. $L A D$, Left atrial diameter; $L A V I$, left atrial valve diameter; $T G F-\beta 1$, transforming growth factor $\beta 1$; $A N P$, atrial natriuretic peptide.

significantly lower in the AF group than in the SR group, and the collagen volume fraction correlated negatively with plasma ANP levels. In this study we found that the patients with AF persistence had higher messenger RNA expressions of collagen III $\alpha$ and lower mRNA expressions of ANP than the patients with SR.

Previous studies ${ }^{21,22}$ have demonstrated that in patients with mitral valve disease who are undergoing the surgical maze procedure for $\mathrm{AF}$, the sinus conversion rates decrease as the preoperative left atrial dimensions increase, and the preoperative left atrial area was a determinant of sinus conversion by using the radiofrequency maze procedure for patients with permanent $\mathrm{AF}$ and mitral valve disease. We have similar results, and the left atrial dimension and the left atrial volume index are associated with the persistence of $\mathrm{AF}$ at 1 year after the maze procedure.
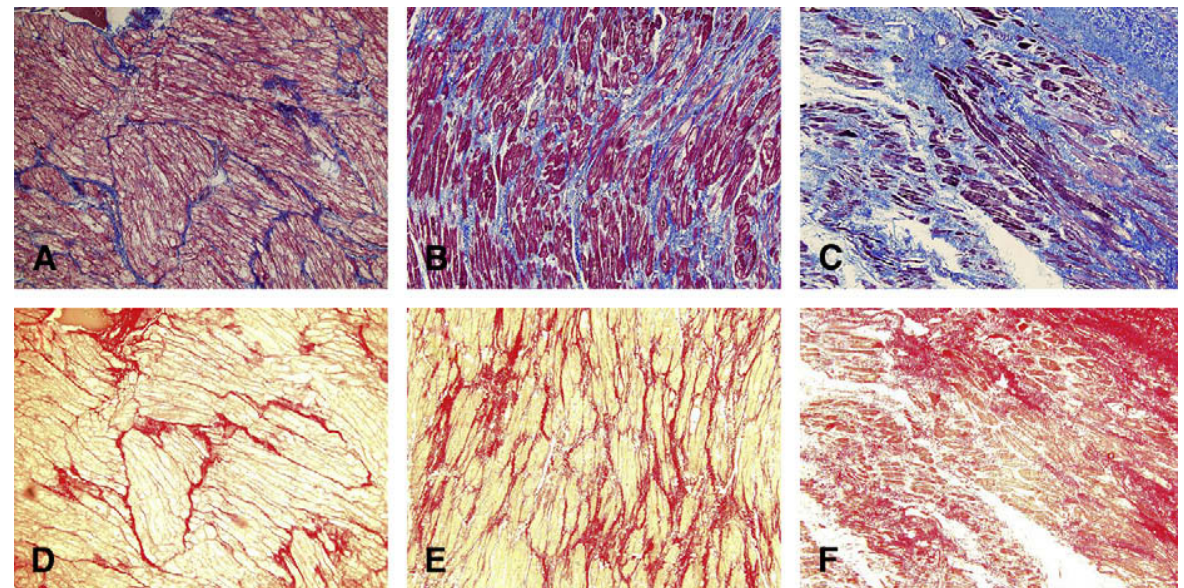

FIGURE 1. Comparisons of the areas of fibrosis in the left atrium among groups. Collagen is stained in blue in Masson trichrome staining (A-C) and red in Sirius red staining (D-F) in the left atrium of the sinus rhythm group (A and D), the atrial fibrillation to sinus rhythm group (B and E), and the atrial fibrillation group $(\mathrm{C}$ and $\mathrm{F})$. (Original magnification $\times 100$.) 


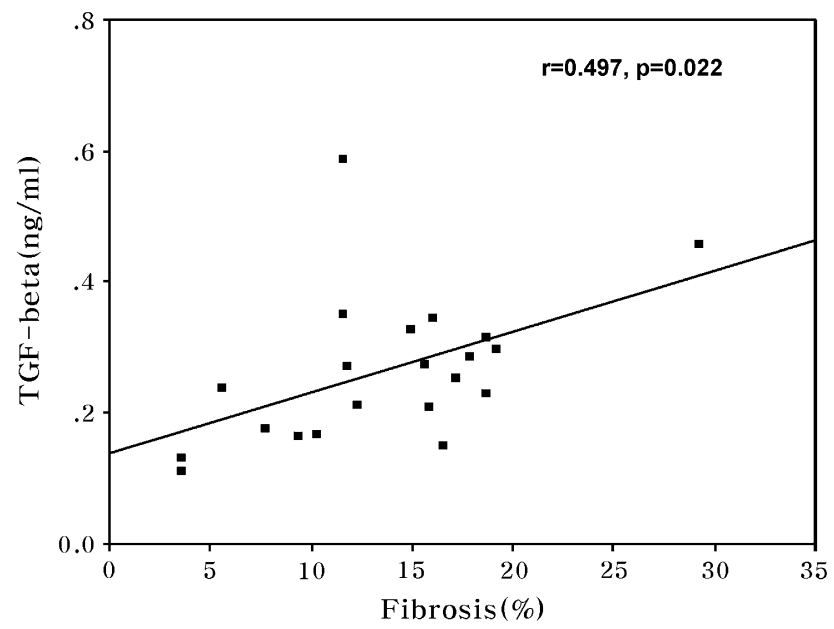

FIGURE 2. Correlation between transforming growth factor $(T G F) \beta 1$ level and the degree of fibrosis. The plasma TGF- $\beta 1$ level was correlated with the degree of fibrosis $(r=0.497, P=.022)$.

C-reactive protein is an acute-phase protein and is an exquisitely sensitive systemic marker of inflammation. C-reactive protein is not only associated with the presence of $\mathrm{AF}$ but might also predict patients at increased risk for future development of $\mathrm{AF}^{23}$

We have some limitations, and small sample size might account for why some of the characteristics were of borderline significance in the multivariable analysis, in particular characteristics that other authors have found to be significant predictors in recurrent $\mathrm{AF}$.

\section{CONCLUSIONS}

Preoperative plasma TGF- $\beta 1$ levels could predict the persistence of AF at 1 year's follow-up in patients who underwent both mitral valvular surgery and the maze procedure with cryoablation because of persistent $\mathrm{AF}$, and preoperative plasma TGF- $\beta 1$ levels were correlated with the degree of fibrosis in the left atria of patients with mitral valvular heart disease.

\section{References}

1. Cox JL, Boineau JP, Schuessler RB, Kater KM, Lappas DG. Five-year experience with the maze procedure for atrial fibrillation. Ann Thorac Surg. 1993;56:814-24.

2. Cox JL, Schuessler RB, Boineau JP. The development of the Maze procedure for the treatment of atrial fibrillation. Semin Thorac Cardiovasc Surg. 2000;12:2-14.

3. Prasad SM, Maniar HS, Camillo CJ, Schuessler RB, Boineau JP, Sundt TM 3rd, et al. The Cox maze III procedure for atrial fibrillation: long-term efficacy in patients undergoing lone versus concomitant procedures. $J$ Thorac Cardiovasc Surg. 2003;126:1822-8.
4. Damiano RJ Jr, Gaynor SL, Bailey M, Prasad S, Cox JL, Boineau JP, et al. The long-term outcome of patients with coronary disease and atrial fibrillation undergoing the Cox maze procedure. J Thorac Cardiovasc Surg. 2003;126: 2016-21.

5. Kosakai Y, Kawaguchi AT, Isobe F, Sasako Y, Nakano K, Eishi K, et al. Cox maze procedure for chronic atrial fibrillation associated with mitral valve disease. J Thorac Cardiovasc Surg. 1994;108:1049-55.

6. Gaynor SL, Schuessler RB, Bailey MS, Ishii Y, Boineau JP, Gleva MJ, et al. Surgical treatment of atrial fibrillation: predictors of late recurrence. J Thorac Cardiovasc Surg. 2005;129:104-11.

7. Yoshihara F, Nishikimi T, Sasako Y, Hino J, Kobayashi J, Minatoya K, et al. Plasma atrial natriuretic peptide concentration inversely correlates with left atrial collagen volume fraction in patients with atrial fibrillation: plasma ANP as a possible biochemical marker to predict the outcome of the maze procedure. $J$ Am Coll Cardiol. 2002;39:288-94.

8. Lamirault G, Gaborit N, Le Meur N, Chevalier C, Lande G, Demolombe S, et al. Gene expression profile associated with chronic atrial fibrillation and underlying valvular heart disease in man. J Mol Cell Cardiol. 2006;40:173-84.

9. Goette A, Juenemann G, Peters B, Klein HU, Roessner A, Huth C, et al. Determinants and consequences of atrial fibrosis in patients undergoing open heart surgery. Cardiovasc Res. 2002;54:390-6.

10. Nakai T, Chandy J, Nakai K, Bellows WH, Flachsbart K, Lee RJ, et al. Histologic assessment of right atrial appendage myocardium in patients with atrial fibrillation after coronary artery bypass graft surgery. Cardiology. 2007;108:90-6.

11. Nattel S, Shiroshita-Takeshita A, Cardin S, Pelletier P. Mechanisms of atrial remodeling and clinical relevance. Curr Opin Cardiol. 2005;20:21-5.

12. Everett TH 4th, Olgin JE. Atrial fibrosis and the mechanisms of atrial fibrillation. Heart Rhythm. 2007;4(suppl):S24-7.

13. Verheule S, Sato T, Everett T, Engle SK, Otten D, Rubart-von der Lohe M, et al. Increased vulnerability to atrial fibrillation in transgenic mice with selective atrial fibrosis caused by overexpression of TGF-beta1. Circ Res. 2004;94: 1458-65.

14. Kupfahl C, Pink D, Friedrich K, Zurbrugg HR, Neuss M, Warnecke C, et al. Angiotensin II directly increases transforming growth factor betal and osteopontin and indirectly affects collagen mRNA expression in the human heart. Cardiovasc Res. 2000;46:463-75.

15. Khan R, Sheppard R. Fibrosis in heart disease: understanding the role of transforming growth factor-beta in cardiomyopathy, valvular disease and arrhythmia. Immunology. 2006; 118:10-24.

16. Grotendorst GR. Connective tissue growth factor: a mediator of TGF-beta action on fibroblasts. Cytokine Growth Factor Rev. 1997;8:171-9.

17. Anne W, Willems R, Roskams T, Sergeant P, Herijgers P, Holemans P, et al. Matrix metalloproteinases and atrial remodeling in patients with mitral valve disease and atrial fibrillation. Cardiovasc Res. 2005;67:655-66.

18. Boldt A, Wetzel U, Lauschke J, Weigl J, Gummert J, Hindricks G, et al. Fibrosis in left atrial tissue of patients with atrial fibrillation with and without underlying mitral valve disease. Heart. 2004;90:400-5.

19. Grammer JB, Bohm J, Dufour A, Benz M, Lange R, Bauernschmitt R. Atrial fibrosis in heart surgery patients Decreased collagen III/I ratio in postoperative atrial fibrillation. Basic Res Cardiol. 2005;100:288-94.

20. Corradi D, Callegari S, Benussi S, Nascimbene S, Pastori P, Calvi S, et al. Regional left atrial interstitial remodeling in patients with chronic atrial fibrillation undergoing mitral-valve surgery. Virchows Arch. 2004;445: 498-505.

21. Chen MC, Chang JP, Chang HW. Preoperative atrial size predicts the success of radiofrequency maze procedure for permanent atrial fibrillation in patients undergoing concomitant valvular surgery. Chest. 2004;125:2129-34.

22. Kosakai Y. Treatment of atrial fibrillation using the Maze procedure: the Japanese experience. Semin Thorac Cardiovasc Surg. 2000;12:44-52.

23. Aviles RJ, Martin DO, Apperson-Hansen C, Houghtaling PL, Rautaharju P, Kronmal RA, et al. Inflammation as a risk factor for atrial fibrillation. Circulation. 2003;108:3006-10. 